\title{
A KR20 and KR21 for Likert Scale Data
}

\author{
Robert C. Foster \\ Bettis Atomic Power Laboratory
}

\begin{abstract}
The traditional KR20 and KR21 estimates of reliability are well known for dichomotous data. This paper derives forms of KR20 and KR21 for polytomous data, which can be applied to Likert scales. The theoretical population reliability is derived for polytomous data which follows the multinomial-Dirichlet model, where responses to each item are multinomial and response category probabilities for each individual follow a Dirichlet distribution. The KR20 and KR21 formulas for polytomous data are constructed as consistent estimators of reliability for this model, and are given in Equations (10) and (11). It is shown that the KR20 for Likert scale data is a linear transformation of Cronbach's alpha. A simulation study comparing the KR20 estimator to several estimators of coefficient omega for unidimensional, tau-equivalent data shows the relative strengths of KR20 as compared to omega: it performs nearly as well for large sample sizes and population reliabilities and is more numerically stable than the estimators based on a factor analysis for small sample sizes and population reliabilities. Furthermore, overfitting a factor analysis model can have serious negatively effects on the performance of reliability estimators based on the model. Lastly, future theoretical development for reliability in multivariate exponential family data are proposed.
\end{abstract}

Keywords - Reliability, KR-20, KR-21, Cronbach's Alpha, Likert Scale, Multinomial, Dirichlet

Robert C. Foster can be contacted at rcfoster@gmail.com. 


\section{Introduction}

Formulas 20 and 21 of Kuder and Richardson (1937), abbreviated throughout this paper as KR20 and KR21, are well-known early estimates for the reliability of a test consisting of dichotomous data. Most test data are not dichotomous, however. Likert scale data (Likert, 1932), in which each response corresponds to one of multiple possible numbered categories (a case of polytomous data) is very commonly used for psychometric scales(Jebb, Ng, \& Tay, 2021). Likert scale data is typically identified as ordinal, but commonly treated as continuous (Wu \& Leung, 2017).

Despite the prevalence of Likert scales, there has not been significant research on estimators specifically for polytomous data, with research instead trending towards more general estimators of reliability based on factorial structures, as in McDonald (1999), or towards ordinal models. Prior to omega, coefficient alpha of Cronbach (1951) was seen and still widely is seen as the general form of KR20 for non-dichotomous data, and is commonly used for Likert scale data. However, Foster (2021) developed KR20 and KR21 formulas for some non-dichotomous data within the exponential family, such as Poisson, exponential, and geometric distributed observations. The traditional dichotomous KR20 can also be derived from the Bernoulli data, which is a member of the exponential family.

The multinomial distribution is a multivariate exponential family distribution equivalent to the Bernoulli distribution for polytomous data. The traditional KR20 and KR21 can be seen as estimators associated with the Bernoulli distribution, so it should therefore be possible to develop similar theory and results for the multinomial distribution which forms the basis of the multiple response categories of the Likert scale. The purpose of this paper, therefore, is to develop an equivalent to KR20 and KR21 for polytomous data based on multivariate exponential family theory. The key idea is this: hierarchical models generate correlation structures. The multinomial-Dirichlet model generates a correlation structure for which the population reliability takes a simple form, and which can be used to represent Likert scale items which are identical in distribution. The KR20 and KR21 estimators of reliability can be derived as consistent estimators of this quantity. The KR20 estimator can also be shown to be a linear transformation of Cronbach's alpha, and so inherits its asymptotic properties. Note that this paper is not concerned with ordinal responses and underlying distributions. The research in this paper does not preclude Likert scale responses as being discretizations of underlying latent constructs, but it is simply not concerned with them. Only the statistical properties of the observed responses is considered.

Section 2 uses probability theory to determine a formula for the reliability of test data when responses to each item are identical and conditionally independent multinomial responses, each of which corresponds to a certain response category label, and response category probabilities for each subject are Dirichlet distributed. Section 3 derives the KR20 and KR21 estimators for polytomous data as consistent estimators of reliability under the multinomial-Dirichlet model, and shows that the KR20 estimate is equal to a linear transformation of Cronbach's alpha. Section 4 conducts a simulation study, using the multinomialDirichlet model to simulate Likert scale test data with a known reliability and comparing the performance of the KR20 estimate of reliability to three different potential estimators of coefficient omega. A discussion of numerical stability and overfitting shows why there may still be a reason to use the KR20 estimate of reliability. 


\section{Theoretical Reliability for the Multinomial-Dirichlet Model}

This section follows the general theoretical development of Foster (2020) and Foster (2021); however, while those papers focused on univariate natural exponential family theory, multivariate exponential family theory is not sufficiently developed so that general statements about reliability for test data following multivariate exponential family distributions can be made. Instead, this paper will focus solely on the multinomial-Dirichlet model, though it is likely that the similar results may be derived for other multivariate exponential family models. For this paper, matrix quantities are denoted in bold and scalar quantities are denoted in standard font. As is standard, random variables are denoted with capital letters and specific realizations of the variable as lower case, though not every capital letter indicates a random variable.

Suppose that the response $\boldsymbol{D}_{i j}$ of subject $i$ to item $j$ on a scale can be modeled as a multinomial distribution of a single trial. Each response is independent and identically distributed conditional on the matrix of category response probabilities $\boldsymbol{\theta}_{i}$, which differs between individuals $i$. Let $L$ be the number of categories in the multinomial distribution, where $L$ is an integer greater than or equal to two. The response probabilities $\theta_{i \ell}$ must sum to 1 over all categories $L$.

$$
\boldsymbol{D}_{i j} \sim \operatorname{Multinomial}\left(\boldsymbol{\theta}_{i}\right) \quad \boldsymbol{\theta}_{i}=\left[\begin{array}{c}
\theta_{i 1} \\
\theta_{i 2} \\
\vdots \\
\theta_{i L}
\end{array}\right]
$$

The outcome of a single trial multinomial random variable is an $L \times 1$ matrix $\boldsymbol{d}_{i j}$ which takes on 1 in category $\ell$ with probability $\theta_{i \ell}$ and returns a 0 in all other categories. An example $\boldsymbol{d}_{i j}$ which returns the second category is

$$
\boldsymbol{d}_{i j}=\left[\begin{array}{c}
0 \\
1 \\
\vdots \\
0
\end{array}\right]
$$

Let each category have unique label $C_{\ell}$, for $\ell=1,2, \ldots, L$, and let $C$ be the $L \times 1$ matrix of category labels.

$$
\boldsymbol{C}=\left[\begin{array}{c}
C_{1} \\
C_{2} \\
\vdots \\
C_{L}
\end{array}\right]
$$

Let $Y_{i j}$ be the response to item $j$ for subject $i$ of a Likert scale. Then $Y_{i j}$ can be defined as

$$
Y_{i j} \mid \boldsymbol{\theta}_{i}=\boldsymbol{C}^{\prime} \boldsymbol{D}_{i j}
$$


The conditional mean and variance of a single response $Y_{i j}$ to item $j$ for subject $i$ given response probabilities $\theta_{i}$ are

$$
\begin{aligned}
E\left[Y_{i j} \mid \boldsymbol{\theta}_{i}\right] & =\boldsymbol{C}^{\prime} \boldsymbol{\theta}_{i} \\
\operatorname{Var}\left(Y_{i j} \mid \boldsymbol{\theta}_{i}\right) & =\boldsymbol{C}^{\prime} V\left(\boldsymbol{\theta}_{i}\right) \boldsymbol{C}
\end{aligned}
$$

where the $L \times L$ covariance matrix $V(\boldsymbol{\theta})$ is is given by

$$
V(\boldsymbol{\theta})=\left[\begin{array}{ccccc}
\theta_{1}\left(1-\theta_{1}\right) & -\theta_{1} \theta_{2} & -\theta_{1} \theta_{3} & \ldots & -\theta_{1} \theta_{L} \\
-\theta_{2} \theta_{1} & \theta_{2}\left(1-\theta_{2}\right) & -\theta_{2} \theta_{3} & \ldots & -\theta_{2} \theta_{L} \\
-\theta_{3} \theta_{1} & -\theta_{3} \theta_{2} & \theta_{3}\left(1-\theta_{3}\right) & \ldots & -\theta_{3} \theta_{L} \\
\vdots & \vdots & \vdots & \ddots & \vdots \\
-\theta_{L} \theta_{1} & -\theta_{L} \theta_{2} & -\theta_{L} \theta_{3} & \ldots & \theta_{L}\left(1-\theta_{L}\right)
\end{array}\right]
$$

The functions $\boldsymbol{C}^{\prime} \boldsymbol{\theta}_{i}$ and $\boldsymbol{C}^{\prime} V\left(\boldsymbol{\theta}_{i}\right) \boldsymbol{C}$ in the mean and variance given in Equation (1) can be written algebraically as

$$
\begin{aligned}
\boldsymbol{C}^{\prime} \boldsymbol{\theta}_{i} & =\sum_{\ell=1}^{L} C_{\ell} \theta_{i \ell} \\
\boldsymbol{C}^{\prime} V\left(\boldsymbol{\theta}_{i}\right) \boldsymbol{C} & =\sum_{\ell=1}^{L} C_{\ell}^{2} \theta_{i \ell}\left(1-\theta_{i \ell}\right)-2 \sum_{\ell_{1}=1}^{L-1} \sum_{\ell_{2}=\ell_{1}+1}^{L} C_{\ell_{1}} C_{\ell_{2}} \theta_{i \ell_{1}} \theta_{i \ell_{2}}
\end{aligned}
$$

The sum score $X_{i}$ for each subject $i$ is defined as the sum of each response $Y_{i j}$ for the subject over all $k$ items.

$$
X_{i}=\sum_{j=1}^{k} Y_{i j}
$$

Because each $Y_{i j}$ is independent and identical conditional on response probabilities $\theta_{i}$, the conditional mean and variance of each sum score $X_{i}$ are

$$
\begin{array}{r}
E\left[X_{i j} \mid \boldsymbol{\theta}_{i}\right]=E\left[\sum_{j=1}^{k} Y_{i j} \mid \boldsymbol{\theta}_{i}\right]=\sum_{j=1}^{k} E\left[Y_{i j} \mid \boldsymbol{\theta}_{i}\right]=k \cdot \boldsymbol{C}^{\prime} \boldsymbol{\theta}_{i} \\
\operatorname{Var}\left(X_{i j} \mid \boldsymbol{\theta}_{i}\right)=\operatorname{Var}\left(\sum_{j=1}^{k} Y_{i j} \mid \boldsymbol{\theta}_{i}\right)=\sum_{j=1}^{k} \operatorname{Var}\left(Y_{i j} \mid \boldsymbol{\theta}_{i}\right)=k \cdot \boldsymbol{C}^{\prime} V\left(\boldsymbol{\theta}_{i}\right) \boldsymbol{C}
\end{array}
$$

Suppose that response probabilities themselves follow a Dirichlet distribution, which is the conjugate prior to the multinomial distribution.

$$
\boldsymbol{\theta}_{i} \sim \operatorname{Dirichlet}\left(\alpha_{1}, \alpha_{2}, \ldots, \alpha_{L}\right)
$$

Each $\alpha_{\ell}$ is a positive real number. Define $M$ as the sum of all $\alpha_{\ell}$ parameters. 


$$
M=\sum_{\ell=1}^{L} \alpha_{\ell}
$$

The mean, variance, and covariance between each element of $\boldsymbol{\theta}_{i}$ are given by

$$
\begin{aligned}
E\left[\theta_{i \ell}\right] & =\frac{\alpha_{\ell}}{M}=\mu_{\ell} \\
\operatorname{Var}\left(\theta_{i \ell}\right) & =\frac{\mu_{\ell}\left(1-\mu_{\ell}\right)}{M+1} \\
\operatorname{Cov}\left(\theta_{i \ell_{1}}, \theta_{i \ell_{2}}\right) & =-\frac{\mu_{\ell_{1}} \mu_{\ell_{2}}}{M+1}
\end{aligned}
$$

Using the law of total expectation and the conditional mean given in Equation (1), the unconditional expectation of response $Y_{i j}$ is

$$
E\left[Y_{i j}\right]=E\left[E\left[Y_{i j} \mid \boldsymbol{\theta}_{i}\right]\right]=E\left[C^{\prime} \boldsymbol{\theta}_{i}\right]=E\left[\sum_{\ell=1}^{L} C_{\ell} \theta_{i \ell}\right]=\sum_{\ell=1}^{L} C_{\ell} \mu_{\ell}
$$

Similarly using the law of total variance and the conditional variance given in Equation (1), the unconditional variance of response $Y_{i j}$ is

$$
\operatorname{Var}\left(Y_{i j}\right)=E\left[\operatorname{Var}\left(Y_{i j} \mid \boldsymbol{\theta}_{i}\right)\right]+\operatorname{Var}\left(E\left[Y_{i j} \mid \boldsymbol{\theta}_{i}\right]\right)=E\left[\boldsymbol{C}^{\prime} V\left(\boldsymbol{\theta}_{i}\right) \boldsymbol{C}\right]+\operatorname{Var}\left(\boldsymbol{C}^{\prime} \boldsymbol{\theta}_{i}\right)
$$

Using Equation (4), the unconditional mean and variance of sum scores $X_{i}$ are then

$$
\begin{aligned}
E\left[X_{i j}\right] & =E\left[E\left[X_{i j} \mid \boldsymbol{\theta}_{i}\right]\right]=k E\left[\boldsymbol{C}^{\prime} \boldsymbol{\theta}_{i}\right] \\
\operatorname{Var}\left(X_{i j}\right) & =E\left[\operatorname{Var}\left(X_{i j} \mid \boldsymbol{\theta}_{i}\right)\right]+\operatorname{Var}\left(E\left[X_{i j} \mid \boldsymbol{\theta}_{i}\right]\right)=k E\left[\boldsymbol{C}^{\prime} V\left(\boldsymbol{\theta}_{i}\right) \boldsymbol{C}\right]+k^{2} \operatorname{Var}\left(\boldsymbol{C}^{\prime} \boldsymbol{\theta}_{i}\right)
\end{aligned}
$$

The goal is to find the correlation $\rho$ between the sum score $X_{i}$ of a subject and the sum score $X_{i}^{*}$ of the subject on a parallel exam. The correlation coefficient is

$$
\rho=\frac{\operatorname{Cov}\left(X_{i}, X_{i}^{*}\right)}{\sqrt{\operatorname{Var}\left(X_{i}\right) \operatorname{Var}\left(X_{i}^{*}\right)}}
$$

Using the law of total covariance, the numerator is equal to

$$
\operatorname{Cov}\left(X_{i}, X_{i}^{*}\right)=E\left[\operatorname{Cov}\left(X_{i}, X_{i}^{*} \mid \boldsymbol{\theta}_{i}\right)\right]+\operatorname{Cov}\left(E\left[X_{i} \mid \boldsymbol{\theta}_{i}\right], E\left[X_{i}^{*} \mid \boldsymbol{\theta}_{i}\right]\right)
$$

Because the $Y_{i j}$ are independent conditional on response probabilities $\theta_{i}$, their sums $X_{i}$ are independent as well. Hence, $E\left[\operatorname{Cov}\left(X_{i}, X_{i}^{*} \mid \boldsymbol{\theta}_{i}\right)\right]=E[0]=0$. Furthermore, $E\left[X_{i} \mid \boldsymbol{\theta}_{i}\right]=$ $E\left[X_{i}^{*} \mid \boldsymbol{\theta}_{i}\right]=\boldsymbol{C}^{\prime} \boldsymbol{\theta}_{i}$, and so $\operatorname{Cov}\left(E\left[X_{i} \mid \boldsymbol{\theta}_{i}\right], E\left[X_{i}^{*} \mid \boldsymbol{\theta}_{i}\right]\right)=\operatorname{Var}\left(k \boldsymbol{C}^{\prime} \boldsymbol{\theta}_{i}\right)=k^{2} \operatorname{Var}\left(\boldsymbol{C}^{\prime} \boldsymbol{\theta}_{i}\right)$.

In the denominator, $\operatorname{Var}\left(X_{i}\right)=\operatorname{Var}\left(X_{i}^{*}\right)=k E\left[\boldsymbol{C}^{\prime} V\left(\boldsymbol{\theta}_{i}\right) \boldsymbol{C}\right]+k^{2} \operatorname{Var}\left(\boldsymbol{C}^{\prime} \boldsymbol{\theta}_{i}\right)$ from Equation (6). Hence, the correlation is equal to

$$
\rho=\frac{k^{2} \operatorname{Var}\left(\boldsymbol{C}^{\prime} \boldsymbol{\theta}_{i}\right)}{k E\left[\boldsymbol{C}^{\prime} V\left(\boldsymbol{\theta}_{i}\right) \boldsymbol{C}\right]+k^{2} \operatorname{Var}\left(\boldsymbol{C}^{\prime} \boldsymbol{\theta}_{i}\right)}=\frac{k}{\left(\frac{E\left[\boldsymbol{C}^{\prime} V\left(\boldsymbol{\theta}_{i}\right) \boldsymbol{C}\right]}{\operatorname{Var}\left(\boldsymbol{C}^{\prime} \boldsymbol{\theta}_{i}\right)}\right)+k}
$$


To calculate $\rho$, the quantities $E\left[\boldsymbol{C}^{\prime} V\left(\boldsymbol{\theta}_{i}\right) \boldsymbol{C}\right]$ and $\operatorname{Var}\left(\boldsymbol{C}^{\prime} \boldsymbol{\theta}_{i}\right)$ must be calculated.

From Equation (3), the quantity $E\left[\boldsymbol{C}^{\prime} V\left(\boldsymbol{\theta}_{i}\right) \boldsymbol{C}\right]$ is equal to

$$
\begin{aligned}
E\left[\boldsymbol{C}^{\prime} V\left(\boldsymbol{\theta}_{i}\right) \boldsymbol{C}\right] & =E\left[\sum_{\ell=1}^{L} C_{\ell}^{2} \theta_{i \ell}\left(1-\theta_{i \ell}\right)-2 \sum_{\ell_{1}=1}^{L-1} \sum_{\ell_{2}=\ell_{1}+1}^{L} C_{\ell_{1}} C_{\ell_{2}} \theta_{i \ell_{1}} \theta_{i \ell_{2}}\right] \\
& =\sum_{\ell=1}^{L} C_{\ell}^{2}\left(E\left[\theta_{i \ell}\right]-E\left[\theta_{i \ell}^{2}\right]\right)-2 \sum_{\ell_{1}=1}^{L-1} \sum_{\ell_{2}=\ell_{1}+1}^{L} C_{\ell_{1}} C_{\ell_{2}} E\left[\theta_{i \ell_{1}} \theta_{i \ell_{2}}\right]
\end{aligned}
$$

Each of these quantities can be calculated directly from the Dirichlet mean, variance, and covariance given in Equation (5).

$$
\begin{gathered}
E\left[\theta_{i \ell}\right]-E\left[\theta_{i \ell}^{2}\right]=E\left[\theta_{i \ell}\right]-\operatorname{Var}\left(\theta_{i \ell}\right)-\left(E\left[\theta_{i \ell}\right]^{2}\right)=\mu_{\ell}\left(1-\mu_{\ell}\right)\left(\frac{M}{M+1}\right) \\
E\left[\theta_{i \ell_{1}} \theta_{i \ell_{2}}\right]=\operatorname{Cov}\left(\theta_{i \ell_{1}}, \theta_{i \ell_{2}}\right)-E\left[\theta_{i \ell_{1}}\right]\left[\theta_{i \ell_{2}}\right]=\mu_{\ell_{1}} \mu_{\ell_{2}}\left(\frac{M}{M+1}\right)
\end{gathered}
$$

The quantity $E\left[\boldsymbol{C}^{\prime} V\left(\boldsymbol{\theta}_{i}\right) \boldsymbol{C}\right]$ is therefore equal to

$$
E\left[\boldsymbol{C}^{\prime} V\left(\boldsymbol{\theta}_{i}\right) \boldsymbol{C}\right]=\frac{M}{M+1}\left(\sum_{\ell=1}^{L} C_{\ell}^{2} \mu_{\ell}\left(1-\mu_{\ell}\right)-2 \sum_{\ell_{1}=1}^{L-1} \sum_{\ell_{2}=\ell_{1}+1}^{L} C_{\ell_{1}} C_{\ell_{2}} \mu_{\ell_{1}} \mu_{\ell_{2}}\right)
$$

Also from Equation (3) and using the Dirichlet mean, variance, and covariance given in Equation (5), the quantity $\operatorname{Var}\left(\boldsymbol{C}^{\prime} \boldsymbol{\theta}_{i}\right)$ is equal to

$$
\begin{aligned}
\operatorname{Var}\left(\boldsymbol{C}^{\prime} \boldsymbol{\theta}_{i}\right) & =\operatorname{Var}\left(\sum_{\ell=1}^{L} C_{\ell} \theta_{i \ell}\right) \\
& =\sum_{\ell=1}^{L} C_{\ell}^{2} \operatorname{Var}\left(\theta_{i \ell}\right)+2 \sum_{\ell_{1}=1}^{L-1} \sum_{\ell_{2}=\ell_{1}+1}^{L} \operatorname{Cov}\left(\theta_{i \ell_{1}}, \theta_{i \ell_{2}}\right) \\
& =\frac{1}{M+1}\left(\sum_{\ell=1}^{L} C_{\ell}^{2} \mu_{\ell}\left(1-\mu_{\ell}\right)-2 \sum_{\ell_{1}=1}^{L-1} \sum_{\ell_{2}=\ell_{1}+1}^{L} C_{\ell_{1}} C_{\ell_{2}} \mu_{\ell_{1}} \mu_{\ell_{2}}\right)
\end{aligned}
$$

Hence, the quantity $E\left[\boldsymbol{C}^{\prime} V\left(\boldsymbol{\theta}_{i}\right) \boldsymbol{C}\right] / \operatorname{Var}\left(\boldsymbol{C}^{\prime} \boldsymbol{\theta}_{i}\right)$ is equal to

$$
\frac{E\left[\boldsymbol{C}^{\prime} V\left(\boldsymbol{\theta}_{i}\right) \boldsymbol{C}\right]}{\operatorname{Var}\left(\boldsymbol{C}^{\prime} \boldsymbol{\theta}_{i}\right)}=\frac{\frac{M}{M+1}\left(\sum_{\ell=1}^{L} C_{\ell}^{2} \mu_{\ell}\left(1-\mu_{\ell}\right)-2 \sum_{\ell_{1}=1}^{L-1} \sum_{\ell_{2}=\ell_{1}+1}^{L} C_{\ell_{1}} C_{\ell_{2}} \mu_{\ell_{1}} \mu_{\ell_{2}}\right)}{\frac{1}{M+1}\left(\sum_{\ell=1}^{L} C_{\ell}^{2} \mu_{\ell}\left(1-\mu_{\ell}\right)-2 \sum_{\ell_{1}=1}^{L-1} \sum_{\ell_{2}=\ell_{1}+1}^{L} C_{\ell_{1}} C_{\ell_{2}} \mu_{\ell_{1}} \mu_{\ell_{2}}\right)}=M
$$


The parallel test reliability, the correlation between sum scores on parallel tests, is therefore equal to

$$
\rho=\frac{k}{M+k}
$$

where $k$ is the test length and $M$ is the sum of all parameters of the Dirichlet distribution of response probabilities $\boldsymbol{\theta}_{i}$.

Equation (8) implies that when the responses conditionally independently follow a multinomial distribution and the distribution of response category probabilities $\boldsymbol{\theta}_{i}$ over all subjects $i$ follows the Dirichlet distribution, the only factors that influence the population reliability of the scale are the test length $k$ and the sum of the Dirichlet parameters $M$. The number of categories $L$ does not matter, nor do the particular category labels, nor do the number of subjects $n$, though these may affect estimators of the reliability, as will be shown in Section 4.

This model is only valid for data which are unidimensional and conditionally independent given each subject $i$. Each item has the same mean response, as non-normal data typically have a mean-variance relation so that different means implies different variances. Further development for non-identical items may be possible, however. Note once again that the model does not preclude the existence of continuous variables underlying probabilities assigned to each category; however, it is not concerned with the shape of the continuous underlying distribution, only that their discretization yields a Dirichlet distribution.

\section{Derivation of KR20 and KR21 Estimators for the Multinomial-Dirichlet Model}

From Equation (7), notice that the $\operatorname{Var}\left(\boldsymbol{C}^{\prime} \boldsymbol{\theta}_{i}\right)$ is closely related to $V(\boldsymbol{\mu})$, where $\boldsymbol{\mu}$ is the $L \times 1$ matrix of mean probabilities of each response category over all subjects for the underlying Dirichlet distribution.

$$
V(\boldsymbol{\mu})=\left[\begin{array}{ccccc}
\mu_{1}\left(1-\mu_{1}\right) & -\mu_{1} \mu_{2} & -\mu_{1} \mu_{3} & \ldots & -\mu_{1} \mu_{L} \\
-\mu_{2} \mu_{1} & \mu_{2}\left(1-\mu_{2}\right) & -\mu_{2} \mu_{3} & \ldots & -\mu_{2} \mu_{L} \\
-\mu_{3} \mu_{1} & -\mu_{3} \mu_{2} & \mu_{3}\left(1-\mu_{3}\right) & \ldots & -\mu_{3} \mu_{L} \\
\vdots & \vdots & \vdots & \ddots & \vdots \\
-\mu_{L} \mu_{1} & -\mu_{L} \mu_{2} & -\mu_{L} \mu_{3} & \ldots & \mu_{L}\left(1-\mu_{L}\right)
\end{array}\right]
$$

Equation (9) is identical to Equation (2), but $\boldsymbol{\mu}$ is used rather than $\boldsymbol{\theta}$ to emphasize that the inputs are mean response category probabilities $\boldsymbol{\mu}$ over all subjects, not mean response category probabilities for individual subject $\boldsymbol{\theta}_{i}$. The relation is given by

$$
(M+1) \operatorname{Var}\left(\boldsymbol{C}^{\prime} \boldsymbol{\theta}_{i}\right)=\boldsymbol{C}^{\prime} V(\boldsymbol{\mu}) \boldsymbol{C} \quad \boldsymbol{\mu}=\left[\begin{array}{c}
\mu_{1} \\
\mu_{2} \\
\vdots \\
\mu_{L}
\end{array}\right]
$$


Using this relation and the fact that $M=E\left[\boldsymbol{C}^{\prime} V\left(\boldsymbol{\theta}_{i}\right) \boldsymbol{C}\right] / \operatorname{Var}\left(\boldsymbol{C}^{\prime} \boldsymbol{\theta}_{i}\right)$, the following is obtained

$$
\begin{aligned}
\frac{k}{k-1}\left(1-\frac{k \cdot C^{\prime} V(\boldsymbol{\mu}) C}{\operatorname{Var}\left(X_{i}\right)}\right) & =\frac{k}{k-1}\left(1-\frac{k(M+1) \operatorname{Var}\left(\boldsymbol{C}^{\prime} \boldsymbol{\theta}_{i}\right)}{\operatorname{Var}\left(X_{i}\right)}\right) \\
& =\frac{k}{k-1}\left(1-\frac{k(M+1) \operatorname{Var}\left(\boldsymbol{C}^{\prime} \boldsymbol{\theta}_{i}\right)}{\operatorname{Var}\left(X_{i}\right)}\right) \\
& =\frac{k}{k-1}\left(1-\frac{k(M+1) \operatorname{Var}\left(\boldsymbol{C}^{\prime} \boldsymbol{\theta}_{i}\right)}{k E\left[\boldsymbol{C}^{\prime} V\left(\boldsymbol{\theta}_{i}\right) \boldsymbol{C}\right]+k^{2} \operatorname{Var}\left(\boldsymbol{C}^{\prime} \boldsymbol{\theta}_{i}\right)}\right) \\
& =\frac{k}{k-1}\left(1-\frac{M+1}{M+k}\right) \\
& =\frac{k}{k-1}\left(\frac{k-1}{M+k}\right) \\
& =\frac{k}{M+k} \\
& =\rho
\end{aligned}
$$

Hence, by Slutsky's theorem, any consistent estimators of the matrix $\boldsymbol{\mu}$ of mean category response probabilities over the entire population and the sum score variance $\operatorname{Var}\left(X_{i}\right)$ may be inserted into right-hand side of the equation

$$
\rho=\frac{k}{k-1}\left(1-\frac{k \cdot \boldsymbol{C}^{\prime} V(\boldsymbol{\mu}) \boldsymbol{C}}{\operatorname{Var}\left(X_{i}\right)}\right)
$$

in order to produce a consistent estimator of reliability $\hat{\rho}$.

For a consistent estimator of the $\operatorname{Var}\left(X_{i}\right)$, the variance of the sum scores, the unbiased sample variance $s_{x}^{2}$ is used.

$$
\operatorname{var}(x)=s_{x}^{2}=\frac{1}{n-1} \sum_{i=1}^{n}\left(x_{i}-\bar{x}\right)^{2}
$$

The quantity $k \cdot \boldsymbol{C}^{\prime} V(\boldsymbol{\mu}) \boldsymbol{C}$ can be estimated in one of two manners.

For the first method, note that by the law of large numbers and the law of total expectation, the proportion of responses in a category $\ell$ for a given test item serves as an estimate of the category mean response $\hat{\mu}_{j, \ell}$

$$
\hat{\mu}_{j \ell}=\frac{\text { Number of responses in category } \ell \text { for item } j}{\text { Number of subjects } \mathrm{n}}
$$

Define $\hat{\boldsymbol{\mu}}_{j}$ as the $L \times 1$ matrix of estimated response category probabilities $\hat{\mu}_{j \ell}$ for item $j$. By the continuous mapping theorem, $\boldsymbol{C}^{\prime} V\left(\hat{\boldsymbol{\mu}}_{j}\right) \boldsymbol{C}$ is a consistent estimator of $\boldsymbol{C}^{\prime} V(\boldsymbol{\mu}) \boldsymbol{C}$. Then a consistent estimator of $k \cdot \boldsymbol{C}^{\prime} V(\boldsymbol{\mu}) \boldsymbol{C}$ is obtained by summing $\boldsymbol{C}^{\prime} V\left(\hat{\boldsymbol{\mu}}_{j}\right) \boldsymbol{C}$ over all $k$ items. This results in the KR-20 formula for Likert data, given by

$$
K R 20=\frac{k}{k-1}\left(1-\frac{\sum_{j=1}^{k} \boldsymbol{C}^{\prime} V\left(\hat{\boldsymbol{\mu}}_{j}\right) \boldsymbol{C}}{s_{x}^{2}}\right)
$$


where $C$ is again the matrix of response categories, $\hat{\boldsymbol{\mu}}_{j}$ is the matrix of estimated response probabilities for each category based only on the responses for test item $j, V\left(\hat{\boldsymbol{\mu}}_{j}\right)$ is Equation $(9)$, and $s_{x}^{2}$ is the sample variance of sum scores $x_{i}$.

Similarly, the proportion of responses in a category $\ell$ for over all test items serves as an estimate of the category mean response $\hat{\mu}_{\ell}$ when all items are identically distributed.

$$
\hat{\mu}_{\ell}=\frac{\text { Number of responses in category } \ell \text { over all items }}{\text { Total number of test responses } n \times k}
$$

Define $\hat{\boldsymbol{\mu}}$ as the $L \times 1$ matrix of estimated response category probabilities $\hat{\mu}_{\ell}$ over all items. Again by the continuous mapping theorem, $k \cdot \boldsymbol{C}^{\prime} V(\hat{\boldsymbol{\mu}}) \boldsymbol{C}$ is a consistent estimator for $k$. $\boldsymbol{C}^{\prime} V(\boldsymbol{\mu}) \boldsymbol{C}$. Hence, The KR21 formula for Likert data is given by

$$
K R 21=\frac{k}{k-1}\left(1-\frac{k \cdot \boldsymbol{C}^{\prime} V(\hat{\boldsymbol{\mu}}) \boldsymbol{C}}{s_{x}^{2}}\right)
$$

where $\boldsymbol{C}$ is again the $L \times 1$ matrix of labels for each response category, $\hat{\boldsymbol{\mu}}$ is the matrix of estimated response probabilities for each category based on all responses for all items, $V(\hat{\boldsymbol{\mu}})$ is again Equation (9), and $s_{x}^{2}$ is the sample variance of sum scores $x_{i}$.

\subsection{The Relationship Between KR-20 and Cronbach's Alpha}

When only $L=2$ categories labeled as $\boldsymbol{C}^{\prime}=\left[\begin{array}{ll}0 & 1\end{array}\right]$ are used, then Equations (10) and (11) reduce to the standard KR20 and KR21 for dichotomous data first introduced in Kuder and Richardson (1937). The KR20 estimate of reliability given in Equation (10) is also closely related to Cronbach's alpha. It can be shown that Equation (10) is simply a linear transformation of Cronbach's alpha when the standard unbiased sample variance is used for $s_{x}^{2}$ and is exactly equal to Cronbach's alpha when the population variance estimate, which divides by $n$ rather than $n-1$, is used.

Let $u_{1 \ell}, u_{2 \ell}, \ldots, u_{n \ell}$ be a sample of size $n$ from a Bernoulli distributed random variable which takes on 1 with probability $\mu_{\ell}$ and 0 with probability $1-\mu_{\ell}$. Let $\hat{\mu}_{\ell}=\bar{u}_{\ell}$ be the estimate of the probability of success, which is just the sample mean. Then because $u_{i \ell}^{2}=u_{i \ell}$ due to the dichotomous nature of the data as only 0 or 1 , the standard unbiased sample variance $s_{u_{\ell}}^{2}$ is algebraically equal to

$$
\begin{aligned}
\operatorname{var}\left(u_{\ell}\right)=\frac{1}{n-1} \sum_{i=1}^{n}\left(u_{i \ell}-\bar{u}_{\ell}\right)^{2} & =\frac{1}{n-1}\left[\left(\sum_{i=1}^{n} u_{i \ell}^{2}\right)-\frac{1}{n}\left(\sum_{i=1}^{n} u_{i \ell}\right)^{2}\right] \\
& =\frac{1}{n-1}\left[\left(\sum_{i=1}^{n} u_{i \ell}\right)-\frac{1}{n}\left(\sum_{i=1}^{n} u_{i \ell}\right)^{2}\right] \\
& =\frac{n}{n-1}\left[\bar{u}_{\ell}-\bar{u}_{\ell}^{2}\right] \\
& =\left(\frac{n}{n-1}\right) \bar{u}_{\ell}\left(1-\bar{u}_{\ell}\right)
\end{aligned}
$$


Similarly, it can be shown that for two samples $u_{\ell_{1}}$ and $u_{\ell_{2}}$ from Bernoulli distributed random variables, the sample covariance is

$$
\operatorname{cov}\left(u_{\ell_{1}}, u_{\ell_{2}}\right)=\left(\frac{n}{n-1}\right)\left[\overline{u_{\ell_{1}} u_{\ell_{2}}}-\bar{u}_{\ell_{1}} \bar{u}_{\ell_{2}}\right]
$$

where $\overline{u_{\ell_{1}} u_{\ell_{2}}}$ is the sample mean of the product of the paired observations from the two samples.

The response $y_{i j}$ to Likert scale item $j$ can be written as a sum of dependent Bernoulli distributed variables with probabilities of success $\mu_{1}, \mu_{2}, \ldots, \mu_{L}$ weighted by response category labels $C_{1}, C_{2}, \ldots, C_{\ell}$. Each Bernoulli variable corresponds to the response being 1 in that particular category and 0 in all others.

$$
y_{i j}=\sum_{\ell=1}^{L} C_{\ell} u_{i \ell}
$$

Remembering that only a single category $\ell$ can be 1 and all others must be 0 , the dependence is mathematically formalized as $\sum_{\ell=1}^{L} \mu_{\ell}=1$ and $u_{i \ell_{1}} u_{i \ell_{2}}=0$ for $\ell_{1} \neq \ell_{2}$. It must therefore be the case that $\overline{u_{\ell_{1}} u_{\ell_{2}}}=0$ and so for the dependent Bernoulli data the relation between the sample covariance and sample means for each category is

$$
\operatorname{cov}\left(u_{\ell_{1}}, u_{\ell_{2}}\right)=-\left(\frac{n-1}{n}\right) \bar{u}_{\ell_{1}} \bar{u}_{\ell_{2}}
$$

Hence, sample variance $s_{y}^{2}$ for the response is equal to

$$
\begin{aligned}
s_{y}^{2}=\operatorname{var}\left(\sum_{\ell=1}^{L} C_{\ell} u_{i \ell}\right) & =\sum_{\ell=1}^{L} C_{\ell}^{2} \operatorname{var}\left(u_{\ell}\right)+\sum_{\ell_{1}=1}^{L-1} \sum_{\ell_{2}=\ell_{1}+1}^{L} 2 C_{\ell_{1}} C_{\ell_{2}} \operatorname{cov}\left(u_{\ell_{1}}, u_{\ell_{2}}\right) \\
& =\left(\frac{n}{n-1}\right)\left[\sum_{\ell=1}^{L} C_{\ell}^{2} \bar{u}_{\ell}\left(1-\bar{u}_{\ell}\right)-2 \sum_{\ell_{1}=1}^{L-1} \sum_{\ell_{2}=\ell_{1}+1}^{L} C_{\ell_{1}} C_{\ell_{2}} \bar{u}_{\ell_{1}} \bar{u}_{\ell_{2}}\right] \\
& =\left(\frac{n}{n-1}\right) C^{\prime} V(\overline{\boldsymbol{u}}) \boldsymbol{C} \\
& =\left(\frac{n}{n-1}\right) \boldsymbol{C}^{\prime} V(\hat{\boldsymbol{\mu}}) \boldsymbol{C}
\end{aligned}
$$

where $V(\hat{\boldsymbol{\mu}})$ is the function in Equation $L \times 1$ matrix of estimated response probabilities for each category, exactly as in the KR20 formula given in Equation (10).

If the data $y_{i j}$ are taken to be the responses from a single item $j$, then $s_{y}^{2}=s_{j}^{2}$ in Equation (10) and this relationship can be inserted directly into the formula for Cronbach's alpha. 


$$
\begin{aligned}
(\text { Cronbach's Alpha }) & =\frac{k}{k-1}\left(1-\frac{\sum_{j=1}^{k} s_{j}^{2}}{s_{x}^{2}}\right) \\
& =\frac{k}{k-1}\left(1-\frac{\frac{n}{n-1} \sum_{j=1}^{k} \boldsymbol{C}^{\prime} V\left(\hat{\boldsymbol{\mu}}_{j}\right) \boldsymbol{C}}{s_{x}^{2}}\right) \\
& =-\frac{k}{(n-1)(k-1)}+\left(\frac{n}{n-1}\right)\left(\frac{k}{k-1}\right)\left(1-\frac{\sum_{j=1}^{k} C^{\prime} V\left(\hat{\boldsymbol{\mu}}_{j}\right) \boldsymbol{C}}{s_{x}^{2}}\right) \\
& =-\frac{k}{(n-1)(k-1)}+\frac{n}{n-1}(K R 20)
\end{aligned}
$$

Solving this equation for KR-20 gives the exact linear transformation between KR-20 and Cronbach's alpha when the unbiased estimate of variance $s_{x}^{2}$ is used.

$$
(\mathrm{KR} 20)=\left(\frac{n-1}{n}\right)(\text { Cronbach's Alpha })+\frac{1}{n}\left(\frac{k}{k-1}\right)
$$

In Equation (12), $n$ is the number of subjects and $k$ is the number of test items.

The linear relationship in Equation (12) implies that as the number of subjects $n$ increases, Cronbach's alpha and the KR20 estimate of reliability given in Equation (10) will converge to equality. KR20 can thus be said to asymptotically inherit the properties of Cronbach's alpha, and should therefore be a consistent estimator of reliability even if the distribution of response categories is some other distribution than Dirichlet.

Equation (12) can also be used to calculate the exact relationship between the bias and variance of the KR-20 estimate of reliabililty and the bias and variance of Cronbach's alpha.

$$
\begin{aligned}
\operatorname{Bias}(\mathrm{KR} 20) & =\left(\frac{n-1}{n}\right) \operatorname{Bias}(\text { Cronbach's Alpha })+\frac{1}{n}\left[\frac{k}{k-1}-\rho\right] \\
\operatorname{Var}(\mathrm{KR} 20) & =\left(\frac{n-1}{n}\right)^{2} \operatorname{Var}(\text { Cronbach'a Alpha })
\end{aligned}
$$

The variance of the KR20 estimator of reliability will always be less than than the variance of Cronbach's Alpha, though the variances will converge as the number of subjects $n$ increases. The bias is slightly more complicated. Cronbach's alpha itself always has a negative bias (Lord, Novick, and Birnbaum (1968), Section 9.3). The effect of using KR20 instead is that the bias is shrunk towards zero and a constant is added. This constant must be positive, as the number of items $k$ is a positive integer so that $k /(k-1)>0$, and the population reliability $\rho<1$ by definition. The exact magnitude of this constant in comparison to the bias of Cronbach's alpha can not be generally calculated, but it can be said that the bias of KR20 is less negative than the bias of Cronbach's alpha (possibly crossing into positive). The two biases also converge to each other as the number of subjects $n$ increases. The result is that KR20 will generally have a smaller mean squared error than Cronbach's alpha for 
small numbers of subjects $n$, and the mean squared error of each will converge to equality as the number of subjects $n$ increases.

Finally, when the population estimate of the variance which divides by $n$ is used rather than the unbiased sample estimate which divides by $n-1$, the KR20 estimate of Equation (10) is algebraically equal to Cronbach's alpha.

$$
\begin{aligned}
\text { (Cronbach's Alpha }) & =\frac{k}{k-1}\left(1-\frac{\sum_{j=1}^{k} s_{j}^{2}}{s_{x}^{2}}\right) \\
& =\frac{k}{k-1}\left(1-\frac{\frac{n}{n-1} \sum_{j=1}^{k} \boldsymbol{C}^{\prime} V\left(\hat{\boldsymbol{\mu}}_{j}\right) \boldsymbol{C}}{s_{x}^{2}}\right) \\
& =\frac{k}{k-1}\left(1-\frac{\sum_{j=1}^{k} \boldsymbol{C}^{\prime} V\left(\hat{\boldsymbol{\mu}}_{j}\right) \boldsymbol{C}}{\frac{n-1}{n} s_{x}^{2}}\right) \\
& =\frac{k}{k-1}\left(1-\frac{\sum_{j=1}^{k} \boldsymbol{C}^{\prime} V\left(\hat{\boldsymbol{\mu}}_{j}\right) \boldsymbol{C}}{\frac{1}{n} \sum_{i=1}^{n}\left(x_{i}-\bar{x}\right)^{2}}\right)
\end{aligned}
$$

Cronbach's alpha itself does not depend on the choice between the two estimates so long the same variance estimate is used consistently in both the numerator and denominator, as either the $1 / n$ or $1 /(n-1)$ will cancel out of both.

\subsection{Example}

Suppose the data in Table 1 from a Likert scale are observed. There are $L=3$ categories, $k=4$ items, and $n=5$ subjects. The response category labels are $\boldsymbol{C}^{\prime}=\left[\begin{array}{lll}1 & 2 & 3\end{array}\right]$. The quantity $s_{x}^{2}$, the unbiased sample variance of the sum scores $x_{i}$, is equal to $s_{x}^{2}=3.3$ for the data in Table 1.

\begin{tabular}{c|cccc|c} 
Subject & Item 1 & Item 2 & Item 3 & Item 4 & Sum \\
\hline A & 1 & 1 & 2 & 1 & 5 \\
B & 3 & 1 & 1 & 2 & 7 \\
C & 2 & 2 & 2 & 2 & 8 \\
D & 3 & 1 & 2 & 2 & 8 \\
E & 3 & 2 & 3 & 2 & 10
\end{tabular}

Table 1: Example Likert scale data used for the calculations in Section 3.2. There are $n=5$ subjects, each of which responded to $k=4$ items. The responses are from a Likert scale with $L=3$ categories, labeled as 1,2 , and 3 .

To calculate the KR20 estimate of reliability for the data in Table 1, first calculate the estimated probabilities of response for each category for each item $j$. For the 
first item, the estimated probabilities for each response category are $\hat{\boldsymbol{\mu}}_{1}=\left[\begin{array}{lll}1 / 5 & 1 / 5 & 3 / 5\end{array}\right]=$ $\left[\begin{array}{lll}0.2 & 0.2 & 0.6\end{array}\right]$. Similarly, $\hat{\boldsymbol{\mu}}_{2}=\left[\begin{array}{lll}0.6 & 0.4 & 0.0\end{array}\right], \hat{\boldsymbol{\mu}}_{3}=\left[\begin{array}{lll}0.2 & 0.6 & 0.2\end{array}\right]$, and $\hat{\boldsymbol{\mu}}_{4}=\left[\begin{array}{lll}0.2 & 0.8 & 0.0\end{array}\right]$. Inserting these into Equation (10), the KR20 estimate of reliability for the data in Table 1 is then

$$
K R 20=\frac{3}{2}\left(1-\frac{\boldsymbol{C}^{\prime} V\left(\hat{\boldsymbol{\mu}}_{1}\right) \boldsymbol{C}+\boldsymbol{C}^{\prime} V\left(\hat{\boldsymbol{\mu}}_{2}\right) \boldsymbol{C}+\boldsymbol{C}^{\prime} V\left(\hat{\boldsymbol{\mu}}_{3}\right) \boldsymbol{C}+\boldsymbol{C}^{\prime} V\left(\hat{\boldsymbol{\mu}}_{4}\right) \boldsymbol{C}}{3.3}\right) \approx 0.752
$$

where $V\left(\hat{\boldsymbol{\mu}}_{j}\right)$ is the estimated mean probability of response for each category using only the data from item $j$ inserted into Equation (9). Note that Cronbach's alpha for the data in Table 1 is equal to 0.606, fitting with the formula given in Equation (12).

To calculate the KR21 estimate of reliability for the data in Table 1, first calculate the estimated probabilities of response for each category over all items in the data set. This is $\hat{\boldsymbol{\mu}}=\left[\begin{array}{lll}6 / 20 & 10 / 20 & 4 / 20\end{array}\right]=\left[\begin{array}{lll}0.3 & 0.5 & 0.2\end{array}\right]$. Using Equation (11), the KR21 estimate of reliability is then

$$
K R 21=\frac{3}{2}\left(1-\frac{4 \cdot \boldsymbol{C}^{\prime} V(\hat{\boldsymbol{\mu}}) \boldsymbol{C}}{3.3}\right) \approx 0.541
$$

where $V(\hat{\boldsymbol{\mu}})$ is the estimated probability of response over all items inserted into Equation (9).

\section{Simulation Study}

Given that the KR20 formula in Equation (10) is equal to a linear transformation of Cronbach's alpha, why use it at all? Recent literature has called for moving away from the use of alpha in favor of of estimators of reliability based on analyses of factorial structures (Hayes \& Coutts, 2020; McNeish, 2018; Trizano-Hermosilla \& Alvarado, 2016). One commonly suggested estimator is coefficient omega "total", hereafter referred to simply as coefficient omega, or simply omega. The primary argument is that at the population level, alpha is only equal to the population reliability $\rho$ for essentially tau equivalent data (McDonald, 1999). Tau-equivalent data are unlikely to occur in practice, however, and more common is congeneric data. Factor analysis based estimators such as omega can accurately estimate the reliability of congeneric data, but alpha underestimates the reliability in such situations. Not all agree, though, with some arguing that alpha still has its uses and that the difference is usually small (Raykov \& Marcoulides, 2019) or simply stating that caution is needed when basing estimates of reliability off of models of an underlying factorial structure (Savalei \& Reise, 2019).

A simulation study can help to show why the KR20 estimate of reliability may still have a place when congeneric estimators such as coefficient omega exist. The multinomialDirichlet model of Section 2 can be used to simulate polytomous, Likert scale data which has a known population reliability $\rho$ in order to test the performance of reliability estimators. The model produces data which are essentially tau equivalent, and so all estimators are equal to the population $\rho$ at the population level. The resulting simulation study therefore compares the statistical performance of each estimator. 


\subsection{Study Design}

The simulation method is as follows.

1. Choose the number of subjects $n$, the number of response categories $L$, and the matrix of labels for each response category $\boldsymbol{C}$.

2. Choose two of the population reliability $\rho$, the number of items $k$, and the sum $M$ of the underlying Dirichlet distribution of response category probabilities for each probability. The three parameters are related by Equation (8), and so the third parameter must be solved for using the chosen values of the first two.

3. Choose parameters $\boldsymbol{\alpha}=\left\{\alpha_{1}, \alpha_{2}, \ldots, \alpha_{L}\right\}$ such that the sum over all $\alpha_{\ell}$ is equal to $M$.

4. Simulate $n$ set of category response probabilities $\boldsymbol{\theta}_{i}$ from a Dirichlet distribution with parameters $\boldsymbol{\alpha}$, one for each subject $i$.

5. For each subject $i$, generate $k$ observations $\boldsymbol{d}_{i j}$ from a multinomial distribution with one trial and response category probabilities given by $\boldsymbol{\theta}_{i}$. Calculate $\boldsymbol{C}^{\prime} \boldsymbol{d}_{i j}$ as the Likert scale response to each item.

This method generates Likert scale data which is unidimensional and tau-equivalent, with population reliability $\rho$. Each item is identical in distribution to all other items.

For the response categories, $L=7$ categories were chosen. Previous research (Leung, 2011; Lozano, García-Cueto, \& Muñiz, 2008) has indicated that the number of categories does not significantly affect the reliability when the number of response categories is moderate, and mathematical theory in Section 2 indicates that changing the number of response categories does not affect the reliability so long as the sum of the parameters of the underlying Dirichlet distribution of response category probabilities $M$ remains constant. Seven was therefore chosen as then number of categories categories was to mirror common Likert scales in practice. Standard labels $\boldsymbol{C}^{\prime}=\left[\begin{array}{llllll}1 & 2 & 3 & 4 & 5 & 6\end{array}\right]$ were used. As shown in Section 2, the category labels do not affect the reliability.

The three variables manipulated were the number of subjects $n$, the number of items $k$, and the population reliability $\rho$. Each was assigned to one of two levels for a complete $2^{3}$ factorial experiment. For the number of subjects $n$, experimental levels of $n=50$ and $n=500$ were used, representing small and large values. For the test length, levels of $k=5$ and $k=20$ items were considered. For the population reliability $\rho$, large and small levels of $\rho=0.3$ and $\rho=0.8$ were used.

For each $(k, \rho)$ combination, the required value of $M$ was calculated as

$$
M=\left(\frac{1-\rho}{\rho}\right) k
$$

This implies that $M$ is unique for each $(k, \rho)$ combination, limiting the ability to compare directly across levels of $\rho$ or directly across levels of $k$. However, preliminary simulations indicated that the the particular of $M$ was by far less important than the test length $k$ or the resulting reliability $\rho$. 
The distribution of the $\boldsymbol{\theta}_{i}$ was set to

$$
\boldsymbol{\theta}_{i} \sim \operatorname{Dirichlet}\left(\frac{M}{16}, \frac{2 M}{16}, \frac{3 M}{16}, \frac{4 M}{16}, \frac{3 M}{16}, \frac{2 M}{16}, \frac{M}{16}\right)
$$

This set of parameters sums to $M$ and shows the mean probability of response in each category over the entire population $\mu_{\ell}$ given by the value of the fraction of $M$ for each parameter. This fraction is either $\mu_{\ell}=1 / 16,2 / 16,3 / 16$, or $4 / 16$, depending on the category. This distribution was chosen as a reasonable distribution which attempted to roughly model a distribution which tended to have the largest probability of response in the middle of the scale, though the ordering of the categories does not matter for the purposes of Equations (10) or (11). In preliminary simulations, the particular configuration of any Dirichlet parameters which summed to $M$ did not significantly affect the estimation of reliability so long as most of the total probability of response was not extremely concentrated in one or two categories.

Four possible estimators of reliability were considered, comprising three possible estimates of coefficient omega and the KR20 estimate of reliability.

1. $\omega_{R 3}$, "Revelle's" omega total as calculated by the default omega() command from the psych package (Revelle, 2021), which uses three group factors.

2. $\omega_{R 1}$, "Revelle's" omega total as calculated by the omega() command from the psych package (Revelle, 2021) but with only one group factor specified.

3. $\omega_{F 1}$, omega total calculated from a one-factor model fit using the factanal() command in base $\mathrm{R}$ (R Core Team, 2021). The factor loadings $\lambda_{j}$ and the uniquenesses $e_{j}$ are extracted and inserted into the formula

$$
\omega_{F 1}=\frac{\left(\sum_{j=1}^{k} \lambda_{j}\right)^{2}}{\left(\sum_{j=1}^{k} \lambda_{j}\right)^{2}+\sum_{j=1}^{k} e_{j}}
$$

This estimator uses a maximum likelihood method which explicitly assumes normality of uniquenesses.

4. KR20, the estimate of reliability for polytomous data given by Equation (10)

"Revelle's" omega total applies a Schmid-Leiman rotation to the factor solution and is recommended in McNeish (2018), where more technical details of the estimator may be found. Cronbach's alpha was not considered because it is a simple linear transformation of KR20 as shown in Section 3.1, and the estimate of coefficient omega produced by the ci.reliability() function from the MBESS package (Kelley, 2021) was considered but judged to be too slow and numerically unstable for certain parameter combinations. Other estimators of reliability such as the greatest lower bound, or variations of coefficient omega such as 
"hierarchical" omega were not considered to keep the focus of the simulation study primarily on the comparison between coefficient omega and KR20. If a researcher is interested, future simulation studies may compare other estimators of reliability.

Each estimator $\hat{\rho}$ was judged using the root mean squared error, bias, and standard error, as calculated using the following formulas.

$$
\begin{aligned}
\operatorname{RMSE}(\hat{\rho}) & =\sqrt{\frac{1}{N_{\text {sims }}} \sum_{s=1}^{N_{\text {sims }}}\left(\hat{\rho}_{s}-\rho\right)^{2}} \\
\operatorname{Bias}(\hat{\rho}) & =\frac{1}{N_{\text {sims }}} \sum_{s=1}^{N_{\text {sims }}}(\hat{\rho}-\rho) \\
S D(\hat{\rho}) & =\sqrt{\frac{1}{N_{\text {sims }}-1} \sum_{s=1}^{N_{\text {sims }}}\left(\hat{\rho}_{s}-\overline{\hat{\rho}}\right)^{2}}
\end{aligned}
$$

A total of one million simulated data sets were generated for each of the eight experimental combinations of $\rho, k$, and $n$. Results are reported to three decimal places. A $99 \%$ empirical bootstrap interval was created for each calculated quantity for all sets of parameters, and the range of all intervals was either 0 or 0.001 when rounded to three decimal places, indicating that three decimal places of accuracy is justified and the Monte Carlo error remaining in the estimates is approximately equivalent to rounding error.

\subsection{Study Results and Discussion}

The results of the simulation study are shown in Table 2 . These results clearly indicate that, in agreement with Savalei and Reise (2019), there is a price to pay for overfitting the model. The estimator $\omega_{R 3}$ of coefficient omega which assumes three factors has the worst root mean squared error for every single set of parameters. It is also positively biased, sometimes strongly positively biased, for every set of parameters except one. This estimate of reliability consistently performs worse than the other estimators of coefficient omega $\omega_{R 1}$ and $\omega_{F 1}$ which correctly specify a single factor and worse than the KR20 estimate of reliability.

The story extends beyond overfitting, however. Suppose a researcher takes the advice of Savalei and Reise (2019) and Yang and Green (2010) and conducts an exploratory factor analysis to ensure that their data are unidimensional before estimating reliability. As seen in both the results shown in Table 2 and the results of the simulation study in Yang and Green (2010), estimators of factorial structures may be numerically unstable when the population reliability $\rho$ is small. Because of this instability, the technical details of how an estimator fits a factorial structure can have a significant effect on the resulting properties of the reliability estimate. For example, in the first set of parameters of Table 2, with $\rho=0.3, k=5$, and $n=50$, both single-factor estimators $\omega_{R 1}$ and $\omega_{F 1}$ have the same root mean squared error rounded to three decimal places. Yet this hides the fact that $\omega_{R 1}$ has higher bias but lower variance, while $\omega_{F 1}$ has lower bias but higher variance. Not even the 
KR20 estimate of Equation (10) is immune from numerical issues - it generally performs worse when $\rho=0.3$ and, as shown in Section 3.1, the act of choosing the unbiased or biased estimate of the sample variance in the denominator affects the resulting properties of the KR20 estimate of reliability. KR20 is generally more numerically stable, however. Increasing either the number of subjects $n$ or the number of items $k$ decreases the RMSE of KR20, but increasing the number of items $k$ from 5 to 20 when $\rho=0.3$ and $n=50$ actually makes the RMSE of $\omega_{R 3}, \omega_{R 1}$, and $\omega_{F 1}$ worse! Keeping the population reliability the same value but increasing the number of items implies that each item itself has a lower factor loading, and lower factor loadings tend to be more difficult to estimate. Why then is KR20 more numerically stable when $k=20$ ? It does not have to estimate factor loadings, because it does not assume a factorial structure at all. Despite being commonly seen as a special case of coefficient omega when factor loadings are equal, the classical test theory model from which Cronbach's alpha and KR20 are derived is in fact more general than the factor model.

This additional generality perhaps explains why when $\rho=0.8$ or $n=500$, the singlefactor estimators of coefficient omega $\omega_{R 1}$ and $\omega_{F 1}$ do tend to have a lower RMSE than KR20. Both estimators of coefficient omega and KR20 converge towards the same RMSE, bias, and standard deviation as $k$ and $\rho$ increase. KR20 consistently shows a small to zero negative bias for every combination of parameters, however, while $\omega_{R 1}$ and $\omega_{F 1}$ can have either a positive or negative bias (though KR20 achieves this negative bias by occasionally producing a negative estimate of reliability, which is not possible for the estimators of coefficient omega considered). In the event that the data are congeneric, KR20 will have a negative bias, though Raykov (1997) indicates that said negative bias may not necessarily be large (though is likely to be biggest when $\rho$ is small, again displaying instability for small population reliabilities). Is trading a few points of RMSE worth guaranteeing that an estimate of reliability will not be positively biased? If the goal is simply to determine whether the reliability is above a certain threshold, it very well might be a worthwhile trade, even when the data are congeneric. However, only the researcher can answer this question. Conversely, if the goal is to investigate the underlying factorial structure of the data, an estimator of coefficient omega such as $\omega_{R 1}$ or $\omega_{F 1}$ would almost certainly be more appropriate.

The purpose of this simulation study is not to recommend one particular estimator of reliability over another but to emphasize that any method of estimating reliability carries with tradeoffs, potential risks, and potential benefits. The primary advantage of coefficient omega over Cronbach's alpha and KR20 is fairly well understood: when essential tau equivalence is violated, alpha underestimates reliability at the population level, but coefficient omega does not. The risks and tradeoffs of coefficient omega and other alternative estimators of reliability are not at all well understood in comparison. There very well may be many scenarios, possibly even most scenarios, where coefficient omega is a far more appropriate estimator of reliability than alpha or KR20. However, no estimator should be used blindly or uniformly. There are always issues beyond model assumptions, of the statistical and numerical properties of the estimator and how those properties align with the questions a researcher is attempting to answer. There is, unfortunately, no single formula which can alleviate the necessity of thinking deeply about the structure of the data, the goals of the analysis, and the possible tradeoffs a researcher is willing to make to achieve those goals. 


\section{$5 \quad$ Future Research Direction and Conclusions}

When responses to a scale can be modeled as a multinomial distribution with known category labels, and the distribution of response category probabilities can be modeled as a Dirichlet distribution, the parallel-test reliability takes on the form of Equation (8)

$$
\rho=\frac{k}{M+k}
$$

which is only affected by the test length $k$ and the sum $M$ of the parameters of the underlying Dirichlet distribution. The KR20 and KR21 estimators of reliability in Equations (10) and (11) can be derived as a model-specific consistent estimators of this quantity. Being either a linear transformation which asymtptotically converges to Cronbach's alpha, or algebraically equal if the biased estimate of the sample variance of sum scores is used, however, the KR20 estimate inherits all the asymptotic properties of alpha for polytomous data. Hence, though the derivation in Section 3 assumes that the distributions of response category probabilities follow a Dirichlet distribution, the KR20 estimate is also a consistent estimator of reliability for other distributions.

Perhaps more importantly, the simulation study of Section 4 shows that there may still be reasons to use KR20 when estimating reliability for polytomous, Likert scale data. It is a simple estimator which is more general than the factor model, and which is generally more numerically stable than estimators of coefficient omega when the population reliability $\rho$ is low and the number of test items $k$ is high, corresponding to low factor loadings in a factor model. It also maintains a small negative bias, which may be useful when the goal is to determine if a reliability is above a threshold rather than determine its exact value. Coefficient omega tends towards overestimation in such scenarios, though the bias can be negative as well (but this typically occurs in situations where both KR20 and omega perform similarly). Even if thresholding the reliability of a scale is not the goal, the use of models based off of factorial structures also carry with them model-based risks of either poor estimation techniques, under estimation, or over estimation of the structure, any of which may make performance worse than KR20.

As stated, Foster (2020) derives much of the theory in Section 2 for the univariate natural exponential family. The theory for multinomial-Dirichlet model works in almost exactly the same way, yet there is little multivariate exponential family theory to exploit in order to prove more general cases. However, based on an assumed correspondence between univariate and multivariate exponential family theory, the author makes the following theoretical predictions:

1. There should exist multivariate analogues of common univariate exponential family distributions, and corresponding multivariate prior distributions which are conjugate to the mean-parameterized multivariate exponential family distributions. Some of these are known, such as the multivariate normal distribution (which serves as its own conjugate prior distribution) and the multinomial distribution with conjugate prior Dirichlet distribution. Others should require more theoretical development. For example, there should exist some form of a multipoisson distribution for which a multivariate gamma distribution is conjugate, and similarly a multivariate inverse gamma distribution which is conjugate to the multivariate gamma distribution. Some 
of these may require unusual parameterizations, or assuming some parameters are known.

2. If a scale consists of independent, identical items for which the response to each item can be seen as a linear combination of the components of a realization of one of these multivariate exponential family distributions with multivariate conjugate prior assumed for the distribution of parameters, the parallel test reliability will be of the same form as Equation (8) for some parameter $M$ of the underlying conjugate prior distribution of parameters, and which is closely related to the shrinkage coefficient for each of the individual parameters in the conjugate posterior distribution for the parameters.

3. There should exist corresponding KR20 and KR21 equations for each multivariate exponential family distribution, and should be similar to the generalized KR20 and KR21 of Foster (2021) and Equations (10) and (11), but should not be simple linear transformations of Cronbach's alpha, as was for the case for KR20 in Section 3.1.

The development of such models and estimators is useful not only for the formulas that can be derived from them, but for new frameworks for simulating data, and may be useful for Bayesian analyses which build hierarchical models to emulate a covariance structure. The general idea of estimating variances using sample means and mean-variance relationships may also be applied to factor analysis models to improve efficiency of esimation. Indeed, the relatively strong performance of coefficient omega for large reliabilities in the simulation study of Section 4, which tests the estimators on polytomous data generated from non-normal distributions, suggests there is still much to learn about factor analysis models themselves. It is clear there is still significant and important research to accomplish in the area of covariance structures and reliabilities for non-normal parametric models. 


\begin{tabular}{|c|c|c|c|c|c|}
\hline Parameters & & $\omega_{R 3}$ & $\omega_{R 1}$ & $\omega_{F 1}$ & $K R 20$ \\
\hline$\rho=0.3$ & RMSE & 0.246 & 0.143 & 0.143 & 0.176 \\
\hline$n=50$ & Bias & 0.217 & 0.120 & 0.050 & -0.012 \\
\hline$k=5$ & $\mathrm{SD}$ & 0.116 & 0.077 & 0.133 & 0.176 \\
\hline$\rho=0.3$ & RMSE & 0.264 & 0.235 & 0.185 & 0.157 \\
\hline$n=50$ & Bias & 0.258 & 0.234 & -0.114 & -0.015 \\
\hline$k=20$ & SD & 0.054 & 0.046 & 0.146 & 0.156 \\
\hline$\rho=0.3$ & RMSE & 0.083 & 0.049 & 0.050 & 0.052 \\
\hline$n=500$ & Bias & 0.056 & 0.006 & 0.005 & -0.001 \\
\hline$k=5$ & SD & 0.061 & 0.049 & 0.049 & 0.052 \\
\hline$\rho=0.3$ & RMSE & 0.061 & 0.044 & 0.070 & 0.046 \\
\hline$n=500$ & Bias & -0.005 & 0.006 & -0.021 & -0.001 \\
\hline$k=20$ & $\mathrm{SD}$ & 0.060 & 0.044 & 0.067 & 0.046 \\
\hline$\rho=0.8$ & RMSE & 0.072 & 0.057 & 0.058 & 0.059 \\
\hline$n=50$ & Bias & 0.055 & -0.002 & -0.004 & 0.000 \\
\hline$k=5$ & SD & 0.046 & 0.057 & 0.058 & 0.059 \\
\hline$\rho=0.8$ & RMSE & 0.054 & 0.046 & 0.053 & 0.047 \\
\hline$n=50$ & Bias & 0.026 & -0.003 & -0.008 & -0.004 \\
\hline$k=20$ & $\mathrm{SD}$ & 0.048 & 0.045 & 0.053 & 0.047 \\
\hline$\rho=0.8$ & RMSE & 0.028 & 0.017 & 0.017 & 0.017 \\
\hline$n=500$ & Bias & 0.018 & 0.000 & 0.000 & 0.000 \\
\hline$k=5$ & SD & 0.022 & 0.017 & 0.017 & 0.017 \\
\hline$\rho=0.8$ & RMSE & 0.017 & 0.014 & 0.014 & 0.014 \\
\hline$n=500$ & Bias & 0.010 & 0.000 & 0.000 & 0.000 \\
\hline$k=20$ & SD & 0.013 & 0.014 & 0.014 & 0.014 \\
\hline
\end{tabular}

Table 2: Simulation Study Results. The parameters are the population reliability $\rho$, the number of subjects $n$, and the number of items $k$. The estimators are $\omega_{R 3}$, omega total as calculated by the default omega () command from the psych package using three factors, $\omega_{R 1}$, omega total as calculated by the default omega() but with only one factor specified, $\omega_{F 1}$, omega total calculated using factor loadings and uniquenesses directly from a one-factor model fit by the base $\mathrm{R}$ factanal() command, and the KR20 estimate of reliability given by Equation (10). The relationship between the root mean squared error (RMSE), bias, and standard deviation (SD) of the estimators for a particular combination of parameters is $R M S E^{2}=$ Bias $^{2}+S D^{2}$. For $\omega_{R 3}$ with $\rho=0.3, n=50$, and $k=5$, for example, $0.246^{2}=0.217^{2}+0.116^{2}$. Each value is calculated from one million simulated data sets. 


\section{References}

Cronbach, L. J. (1951, September). Coefficient alpha and the internal structure of tests. Psychometrika, $16(3), 297-334$.

Foster, R. C. (2020, June). A generalized framework for classical test theory. The Journal of Mathematical Psychology, 96.

Foster, R. C. (2021, February). Kr20 and kr21 for some nondichotomous data (it's not just cronbach's alpha). Educational and Psychological Measurement.

Hayes, A. F., \& Coutts, J. J. (2020). Use omega rather than cronbach's alpha for estimating reliability. but.... Communication Methods and Measures, 14(1), $1-24$.

Jebb, A. T., Ng, V., \& Tay, L. (2021). A review of key likert scale development advances: 1995-2019. Frontiers in Psychology, 12.

Kelley, K. (2021). Mbess: The mbess $\mathrm{r}$ package [Computer software manual]. Retrieved from https://CRAN.R-project.org/package=MBESS (R package version 4.8.1)

Kuder, G. F., \& Richardson, M. W. (1937). The theory of the estimation of test reliability. Psychometrika, 2(3), $151-160$.

Leung, S.-O. (2011). A comparison of psychometric properties and normality in 4-, 5-, 6-, and 11-point likert scales. Journal of Social Service Research, 37(4), $412-421$.

Likert, R. (1932). A technique for the measurement of attitudes. New York.

Lord, F. M., Novick, M. R., \& Birnbaum, A. (1968). Statistical theories of mental test scores. Oxford, England: Addison-Wesley.

Lozano, L. M., García-Cueto, E., \& Muñiz, J. (2008). Effect of the number of response categories on the reliability and validity of rating scales. Methodology, 4 (2), 73-79.

McDonald, R. (1999). Test theory: A unified treatment. Taylor \& Francis.

McNeish, D. (2018). Thanks coefficient alpha, we'll take it from here. Psychological Methods, 23(3), 412-433.

R Core Team. (2021). R: A language and environment for statistical computing [Computer software manual]. Vienna, Austria. Retrieved from https://www.R-project.org/

Raykov, T. (1997). Scale reliability, cronbach's coefficient alpha, and violations of essential tau-equivalence with fixed congeneric components. Multivariate Behavioral Research, 32(4), 329-353.

Raykov, T., \& Marcoulides, G. A. (2019). Thanks coefficient alpha, we still need you! Educational and Psychological Measurement, 79(1), 200-210.

Revelle, W. (2021). psych: Procedures for psychological, psychometric, and personality research [Computer software manual]. Evanston, Illinois. Retrieved from https : //CRAN. R-project.org/package=psych (R package version 2.1.9)

Savalei, V., \& Reise, S. P. (2019). Don't forget the model in your model-based 
reliability coefficients: A reply to mcneish (2018). Collabra:Psychology, 5(1).

Trizano-Hermosilla, I., \& Alvarado, J. (2016, 05). Best alternatives to cronbach's alpha reliability in realistic conditions: Congeneric and asymmetrical measurements. Frontiers in Psychology, 7.

Wu, H., \& Leung, S.-O. (2017). Can likert scales be treated as interval scales? - a simulation study. Journal of Social Service Research, 43(4), 527-532.

Yang, Y., \& Green, S. B. (2010). A note on structural equation modeling estimates of reliability. Structural Equation Modeling: A Multidisciplinary Journal, 17(1), 66-81. 